Check for updates

Cite this: Mater. Adv., 2021, 2,3328

Received 22nd February 2021 Accepted 10th April 2021

DOI: $10.1039 / \mathrm{d} 1 \mathrm{ma} 00154 \mathrm{j}$

rsc.li/materials-advances

\section{Synthesis and superconductivity of new TiNiSi-type equiatomic germanide ThIrGe}

\author{
Guorui Xiao, ${ }^{\text {abc }}$ Qinqing Zhu, ${ }^{\text {bcd }}$ Yanwei Cui, ${ }^{\text {abc }}$ Bin Liu, ${ }^{\text {bc }}$ Jifeng Wu, ${ }^{\text {bc }}$ Baizhuo Li, ${ }^{a}$ \\ Siqi Wu, ${ }^{a}$ Guang-han $\mathrm{CaO}^{\mathrm{a}}$ and Zhi Ren (iD *bc
}

\begin{abstract}
A new germanide ThlrGe has been synthesized and characterized by X-ray diffraction, transmission electron microscopy, electrical resistivity, magnetic susceptibility and thermodynamic measurements. The structural refinement shows that, in contrast to tetragonal ThlrSi, ThlrGe crystallizes in the TiNiSi-type orthorhombic structure with the Pnma space group ( $a=7.2321(2) \AA, b=4.3802(1) \AA$, and $c=7.7107(2) \AA$ ), which is the first Th-based ternary equiatomic intermetallic compound of this structural type. Below $T_{\mathrm{c}}=$ $5.25 \mathrm{~K}$, ThlrGe becomes a weak-coupling type-II superconductor with a fully isotropic superconducting gap. The Sommerfield coefficient, upper critical field, and Ginzburg-Laudau parameter are determined to be $11.8 \mathrm{~mJ} \mathrm{~mol}^{-1} \mathrm{~K}^{-2}, 2.9 \mathrm{~T}$, and 19.9, respectively. First-principles calculations indicate that the density of states at the Fermi level are dominated by a hybridized contribution from the orbitals of Th, Ir and Ge, and are enhanced by the spin-orbit coupling. In addition, we demonstrate that the structural difference between ThlrGe and ThlrSi can be understood in terms of the Gibbs formation energy.
\end{abstract}

\section{Introduction}

Ternary equiatomic intermetallic compounds are a large family of materials and have been studied extensively over the past few decades. ${ }^{1-6}$ These compounds have the general formula $\mathrm{MM}^{\prime} \mathrm{X}$, where $\mathbf{M}$ can be an early transition-metal, a rare-earth or an actinide element, $\mathbf{M}^{\prime}$ is a late transition-metal element, and $\mathrm{X}$ is $\mathrm{Si}$, Ge or P. Depending on the constituent elements, the $\mathrm{MM}^{\prime} \mathrm{X}$ compounds crystallize in a variety of structures, including hexagonal ZrNiAl-type, ${ }^{7}$ orthorhombic TiNiSi-type, ${ }^{8}$ and tetragonal LaPtSi-type ${ }^{9}$ (see Fig. 1). Both the ZrNiAl-type and TiNiSitype structures can be viewed as two-dimensional homologues based on the $\mathrm{AlB}_{2}$-type structure, in which the key structural component is the tricapped trigonal prisms with metal atoms at the corners and non-metal atoms in the center. ${ }^{10}$ The ZrNiAltype structure contains six-membered rings enclosing an isolated prism, while the TiNiSi-type one consists of zigzag sheets of corner-sharing trigonal prisms. ${ }^{11}$ By contrast, the LaPtSi-type structure is noncentrosymmetric and an ordered variant of the $\alpha-\mathrm{ThSi}_{2}$ structure. $^{12}$ In addition to this structural diversity, these ternary compounds exhibit a range of interesting physical

\footnotetext{
${ }^{a}$ Department of Physics, Zhejiang University, Hangzhou 310027, P. R. China

${ }^{b}$ School of Science, Westlake University, 18 Shilongshan Road, Hangzhou 310064, P. R. China.E-mail: renzhi@westlake.edu.cn

Institute of Natural Sciences, Westlake Institute for Advanced Study, 18 Shilongshan Road, Hangzhou 310064, P. R. China

${ }^{d}$ Department of Physics, Fudan University, Shanghai 200433, P. R. China
}

properties, such as complex magnetic ordering, ${ }^{9,13-15}$ heavy fermion behavior ${ }^{16,17}$ and superconductivity. ${ }^{18-24}$

Among the TiNiSi-type $\mathbf{M M}^{\prime} \mathrm{X}$ compounds, the U-based UTGe ( $\mathrm{T}=\mathrm{Co}, \mathrm{Rh}$ and $\mathrm{Ir}$ ) has received considerable attention. $\mathrm{UCoGe}^{19,25}$ and $\mathrm{URhGe}^{20}$ show coexistence of ferromagnetism and superconductivity at ambient pressure. While the underlying mechanism remains unclear, both superconductivity and magnetic order are believed to be due to the $\mathrm{U} 5 \mathrm{f}$ electrons. In comparison, UIrGe orders antiferromagnetically and exhibits no superconductivity. ${ }^{17,26}$ Except for 5 f electrons, Th has the same electronic configuration as U. It is thus of interest to investigate the Th-based isostructural counterparts, which serve as nonmagnetic references to UTGe. However, for the ThTGe series, only ThCoGe has been known to exist and it has an $\mathrm{AlB}_{2}$-type structure. ${ }^{27}$ Moreover, while all of ThTSi members can be synthesized and are found to be superconducting, they adopt the noncentrosymmetric LaPtSi-type structure. ${ }^{28}$ Actually, to our knowledge, no Th-based ternary equiatomic compound with the TiNiSi-type structure has been reported to date.

In this paper, we report the synthesis, crystal structure and physical properties of ThIrGe, a new ternary phase in the Th-IrGe system. It is found that this compound possesses the orthorhombic TiNiSi-type structure and exhibits bulk type-II superconductivity below $5.25 \mathrm{~K}$, which is the highest among ternary equiatomic germanides. Various superconducting parameters such as electron-phonon coupling constant, critical fields, coherence length and penetration depth, are obtained. Theoretical calculations reveal a significant hybridization between the Th, Ir and Ge orbitals at the Fermi level and 
(a)

"ZrNiAl-type"

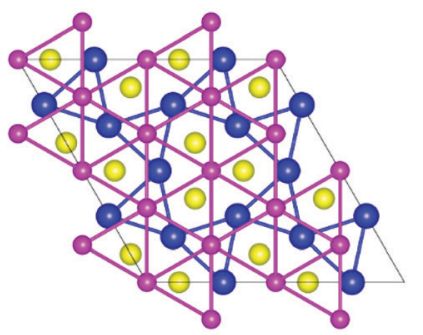

(b)

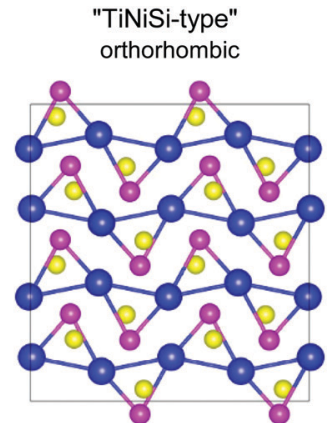

(c)

"LaPtSi-type" tetragonal

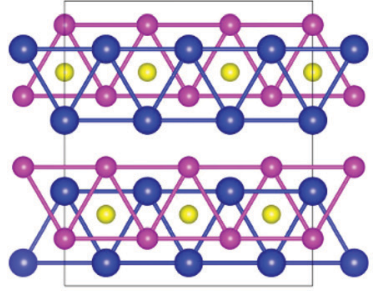

Fig. 1 Schematic structure of (a) hexagonal ZrNiAl-type, (b) orthorhombic TiNiSi-type and (c) tetragonal LaPtSi-type ternary equiatomic intermetallic compounds. The blue, pink, and yellow atoms correspond to $M, M^{\prime}$, and $X$, respectively.

nonnegligible effects of spin-orbit coupling (SOC). In addition, a comparison is made between the Gibbs formation energies of ThIrGe and ThIrSi in both the TiNiSi-type and LaPtSi-type structures.

\section{Materials and methods}

Polycrystalline ThIrGe sample was prepared by the arc-melting method. Stoichiometric amounts of high purity Th (99.5\%), Ir (99.9\%) and Ge (99.99\%) elements were mixed and pressured into a pellet in an argon-filled glove box. The pellet was melted in an arc furnace under high-purity argon atmosphere for several times to ensure homogeneity, followed by rapid cooling on a water-chilled copper plate. The as-cast ingot was sealed into an evacuated quartz tube, annealed at $1000{ }^{\circ} \mathrm{C}$ for 7 days, and then quenched to room temperature. The phase purity was checked by powder X-ray diffraction (XRD) at room temperature using a Bruker D8 Advance diffractometer with $\mathrm{Cu} \mathrm{K} \alpha$ radiation. The crystal structure refinement was carried out using the Rietveld method with the GSAS software. ${ }^{29}$ The chemical composition was measured by the Octane Plus Detector (AMETEX EDAX) coupled to a field-emitting scanning electron microscope (SEM; Hitachi S-4800). The microstructure was examined in a FEI Tecnai G2 F20 S-TWIN transmission electron microscope (TEM) operated at an accelerating voltage of $200 \mathrm{kV}$. Electrical resistivity and specific heat data were measured in a Quantum Design Physical Property Measurement System (PPMS-9 Dynacool). The dc magnetization measurements were performed in a Quantum Design Magnetic Property Measurement System (MPMS3).

First-principles calculations were carried out in the Vienna $\mathrm{Ab}$ initio Simulation Package (VASP) $)^{30}$ based on density functional theory. The Perdew-Burke-Ernzerhof (PBE) ${ }^{31}$ exchange-correlation functional was used and the convergence threshold of HellmannFeynman force is $0.01 \mathrm{eV} \AA^{-1}$. The energy convergence criterion was set to $10^{-6} \mathrm{eV}$ for structural optimization and $10^{-8} \mathrm{eV}$ for other purposes. In both structural optimization and self-consistent calculations, the wavefunction cutoff energy was fixed to $450 \mathrm{eV}$ and the $\Gamma$-centered $k$ mesh was set to $5 \times 8 \times 4$. The latter was increased to $10 \times 16 \times 8$ for density-of-state (DOS) calculations.

\section{Results and discussion}

\subsection{Crystal structure and chemical composition}

Fig. 2 shows XRD patterns of ThIrGe together with its structural refinement profile, and the refined results are listed in Table 1.

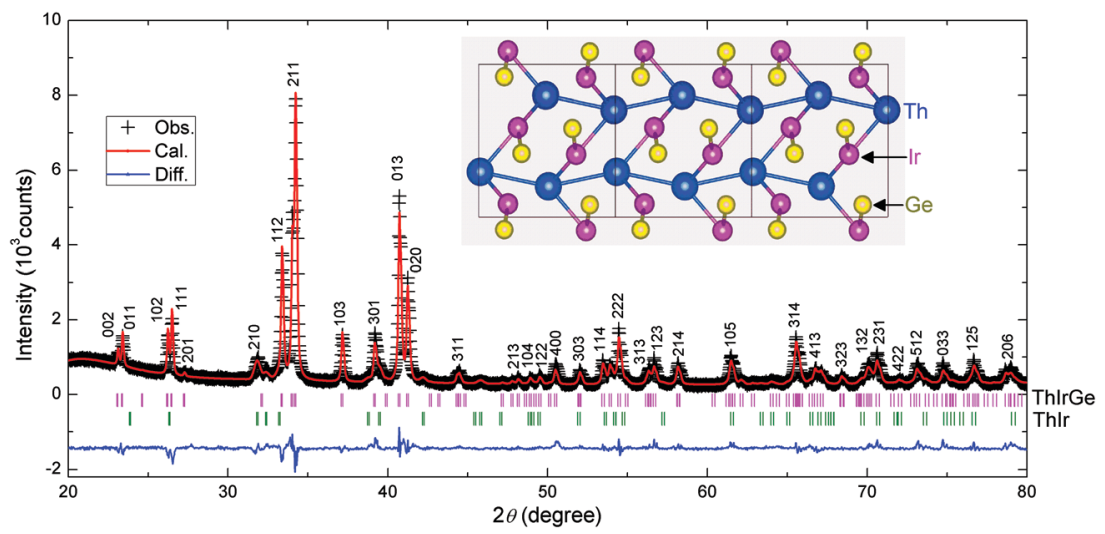

Fig. 2 Powder X-ray diffraction pattern at room temperature together with its structural refinement profile for the ThlrGe sample. The black crosses are the observed patterns and indexed on an orthorhombic unit-cell. The red and blue lines are the calculated pattern and difference plot, respectively. The peak positions for ThlrGe and Thlr are indicated by the ticks. The inset shows a schematic structure of ThlrGe, and the bonds between nearest-neighbour Th-Th, Th-Ir and Ir-Ge atoms are highlighted. 
Except for a few small ones, the diffraction peaks can be well indexed on an orthorhombic lattice with the Pnma space group. This indicates that the main phase of the sample has a TiNiSitype structure, which is used for the structural refinement. There is a satisfactory agreement between the calculated and observed patterns $\left(R_{\mathrm{wp}}=6.5 \%, R_{\mathrm{p}}=4.9 \%, \chi^{2}=2.45\right)$ and a minor impurity phase of ThIr $(\sim 8 \%)$ is also identified. The refined lattice parameters are $a=7.2321(2) \AA, b=4.3802(1) \AA, c=$ 7.7107(2) $\AA$, which yields a unit-cell volume of $244.26(2) \AA^{3}$. In the lattice of ThIrGe, all of Th, Ir and Ge atoms occupy the $(0.0098,0.25,0.7024),(0.2864,0.25,0.4121)$ and $(0.1864$, $0.25,0.0816)$, respectively, all of which are locally noncentrosymmetric. As shown in the inset of Fig. 2, the nearest neighbour Th atoms form zigzag chains running along the $a$-axis with an interatomic distance of $3.689 \AA$, which is slightly larger than that $(3.60 \AA)$ of the sum of thorium atomic radii. A set of selected interatomic distances in ThIrGe structure is given in Table 2. One can note that the nearest neighbour distance between the Th and Ir atom is only $3.002 \AA$. Actually, this Th-Ir distance is even shorter than those of in the cubic Lave phase superconductor $\operatorname{ThIr}_{2}(3.176 \AA)^{32}$ and noncentrosymmetric superconductor ThIrSi (3.138 ̊). ${ }^{28}$ This, together with the short nearest neighbour Th-Ir (3.002 $\mathrm{A})$, and Ir-Ge $(2.557 \AA)$ distances, suggests a hybridization of Th, Ir and Ge orbitals at the Fermi level, which is consistent with the theoretical calculation results shown below.

This crystal structure of ThIrGe is further confirmed by highresolution TEM (HRTEM) measurements on single-crystalline grains, typical results of which are displayed in Fig. 3(a) and (b). As can be seen in Fig. 3(a), the selected-area electron diffraction (SAED) taken along [ $\left[\begin{array}{lll}-2 & 1 & 1\end{array}\right]$ zone axis exhibits a well-defined spot pattern, and the spots near the center can be indexed to the (102), (111) and (213) planes of the TiNiSi-type structure. Moreover, from the HRTEM image of the same area shown in Fig. 3(b), two lattice spacings of $0.327 \mathrm{~nm}$ and $0.340 \mathrm{~nm}$ can be resolved, which correspond well to the (111) and (102) planes, respectively. Note that the angle between these planes is $53^{\circ}$, which is quite as expected in the TiNiSi-type structure. On the other hand, the EDX measurements show that the ThIrGe sample is chemically homogeneous with an average

Table 1 Structural refinement results for ThlrGe

\begin{tabular}{|c|c|c|c|c|c|}
\hline \multicolumn{6}{|l|}{ ThIrGe } \\
\hline \multicolumn{5}{|c|}{ Space group } & Pnma \\
\hline \multirow{7}{*}{\multicolumn{5}{|c|}{$\begin{array}{l}a(\AA) \\
b(\AA) \\
c(\AA) \\
V\left(\AA^{3}\right) \\
R_{\mathrm{wp}} \\
R_{\mathrm{p}} \\
\chi^{2}\end{array}$}} & $7.2321(2)$ \\
\hline & & & & & $4.3802(1)$ \\
\hline & & & & & $7.7107(2)$ \\
\hline & & & & & $244.26(2)$ \\
\hline & & & & & $6.5 \%$ \\
\hline & & & & & $4.9 \%$ \\
\hline & & & & & 2.45 \\
\hline Atoms & $X$ & $y$ & $z$ & Occ. & $U_{\text {iso }}$ \\
\hline Th & $0.0098(5)$ & 0.25 & $0.7024(1)$ & 1 & $0.0064(6)$ \\
\hline Ir & $0.2864(4)$ & 0.25 & $0.4121(3)$ & 1 & $0.0075(1)$ \\
\hline $\mathrm{Ge}$ & $0.1864(7)$ & 0.25 & $0.0816(7)$ & 1 & $0.021(2)$ \\
\hline
\end{tabular}

Table 2 Selected interatomic distances for ThlrGe

\begin{tabular}{llll}
\hline Interaction & Distance $(\AA)$ & Interaction & Distance $(\AA)$ \\
\hline Th-Ge $(\times 2)$ & $3.238(5)$ & Th-Th $(\times 2)$ & $3.816(2)$ \\
Th-Ge $(\times 2)$ & $3.096(5)$ & Th-Th $(\times 2)$ & $3.689(1)$ \\
Th-Ge $(\times 1)$ & $3.190(6)$ & Th-Ir $(\times 1)$ & $3.002(4)$ \\
Th-Ge $(\times 1)$ & $3.205(6)$ & Th-Ir $(\times 1)$ & $3.382(4)$ \\
Ir-Ge $(\times 1)$ & $2.649(5)$ & Th-Ir $(\times 2)$ & $3.189(3)$ \\
Ir-Ge $(\times 1)$ & $2.894(5)$ & Th-Ir $(\times 2)$ & $3.095(3)$ \\
Ir-Ge $(\times 2)$ & $2.557(2)$ & & \\
\end{tabular}
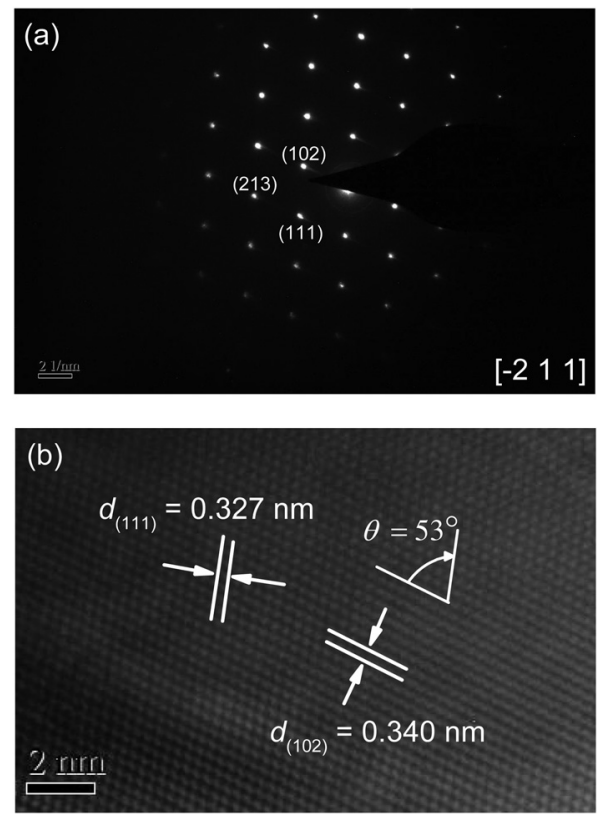

Fig. 3 (a) Selected-area electron diffraction pattern taken along the [ -211 ] zone axis of a single-crystalline grain of the ThlrGe sample. (b) The corresponding high resolution TEM image. The distance and angel between the planes are indicated.

composition of $\mathrm{Th}_{0.9(1)} \operatorname{IrGe}_{1.1(1)}$. This demonstrates that ThIrGe is stoichiometric within the experimental error, in agreement with the refined occupancies.

\subsection{Normal-state and superconducting properties}

The temperature dependence of resistivity $\rho$ under zero field for the ThIrGe sample is shown in Fig. 4(a). With decreasing temperature, $\rho$ decreases smoothly and exhibits a metallic behavior. The residual resistivity $\rho_{0}$ and residual resistivity ratio $\mathrm{RRR}=\rho(300 \mathrm{~K}) / \rho_{0}$ are $\sim 75 \mu \Omega \mathrm{cm}$ and $\sim 3.3$, respectively. Nevertheless, since our sample has a polycrystalline nature, these values depend largely on the contribution of grain boundaries and hence are not a good indicator of the sample quality. On cooling below $5.5 \mathrm{~K}, \rho$ drops abruptly and becomes zero at $\sim 5 \mathrm{~K}$. As shown in Fig. 4(b), the resistive transition is gradually suppressed to lower temperature with increasing the magnetic field. This clearly indicates that the $\rho$ drop is due to a superconducting transition. Here $T_{\mathrm{c}}$ is defined as the midpoint of $\rho$ drop and found to be $5.25 \mathrm{~K}$ at zero field. While this value 

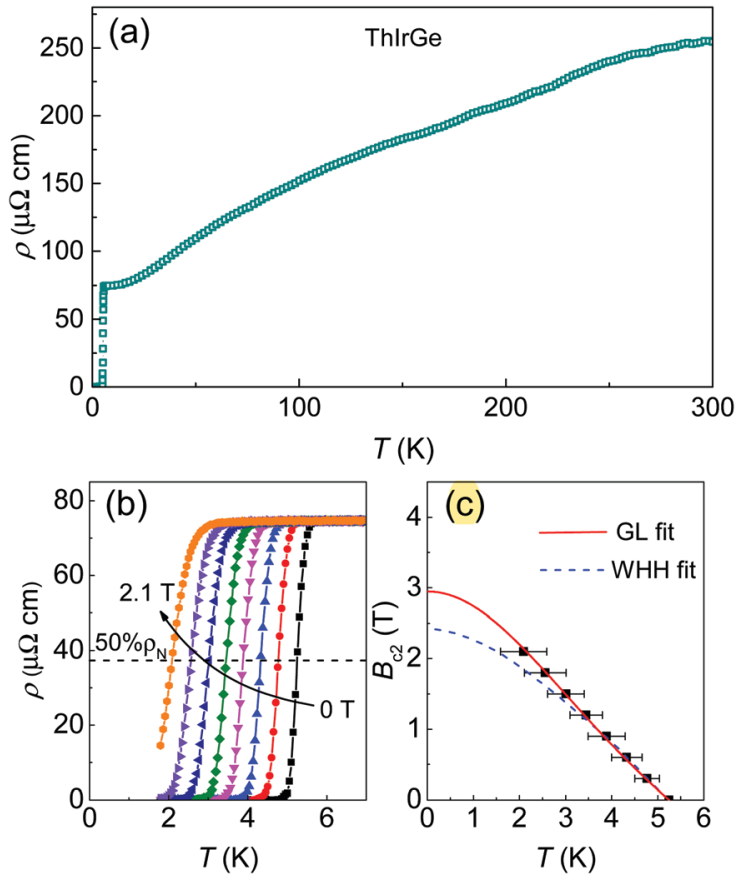

Fig. 4 (a) Temperature dependence of resistivity for the ThlrGe sample. (b) Low temperature resistivity data under various fields from 0 up to $2.1 \mathrm{~T}$ (c) Temperature dependence of the upper critical field with the horizontal error bars denoting the transition width. The red solid line and blue dashed line are GL and WHH fits to the data, respectively.

is close to that of $\operatorname{ThIr}_{2}(5.58 \mathrm{~K}),{ }^{32}$ no corresponding phase is detected in XRD and SEM analysis. In addition, although ThIr impurity is present, its reported $T_{\mathrm{c}}$ is only $0.37 \mathrm{~K} .^{33}$ Thus the observed superconductivity is undoubtedly ascribed to ThIrGe. To our knowledge, this $T_{\mathrm{c}}$ of $5.25 \mathrm{~K}$ is higher than any previously known ternary equiatomic germanides. ${ }^{34}$ Following the same criterion at zero field, the $T_{\mathrm{c}}$ values at various fields are obtained and summarized in the upper critical field $\left(B_{\mathrm{c} 2}\right)$ versus temperature phase diagram displayed in Fig. 4(c), in which the horizontal error bars denote the transition width. Notably, the $B_{\mathrm{c} 2}$ data show deviation from the WerthamerHelfand-Hohenberg curve ${ }^{35}$ at low temperature but follow nicely the Ginzburg-Landau (GL) relationship

$$
B_{\mathrm{c} 2}(T)=B_{\mathrm{c} 2}(0) \frac{1-t^{2}}{1+t^{2}}
$$

where $t=T / T_{\mathrm{c}}$. An extrapolation of the data to $0 \mathrm{~K}$ gives the zerotemperature upper critical field $B_{\mathrm{c} 2}(0)=2.9 \mathrm{~T}$. This value is far below the Pauli limiting field $B_{\mathrm{P}}(0)=1.86 T_{\mathrm{c}} \approx 9.7 \mathrm{~T},{ }^{36}$ suggesting that $B_{\mathrm{c} 2}$ of ThIrGe is limited by the orbital effect.

The occurrence of superconductivity in ThIrGe is corroborated by the magnetic susceptibility $(\chi)$ measurements under a magnetic field of $1 \mathrm{mT}$ in both the zero-field cooling (ZFC) and field cooling (FC) modes, whose results are shown in Fig. 5(a). A strong diamagnetic transition is observed in both the ZFC and FC data, and its onset temperature of $5.22 \mathrm{~K}$ agrees well with the $T_{\mathrm{c}}$ determined from the resistivity measurement. Below the transition, a bifurcation develops between the two curves,
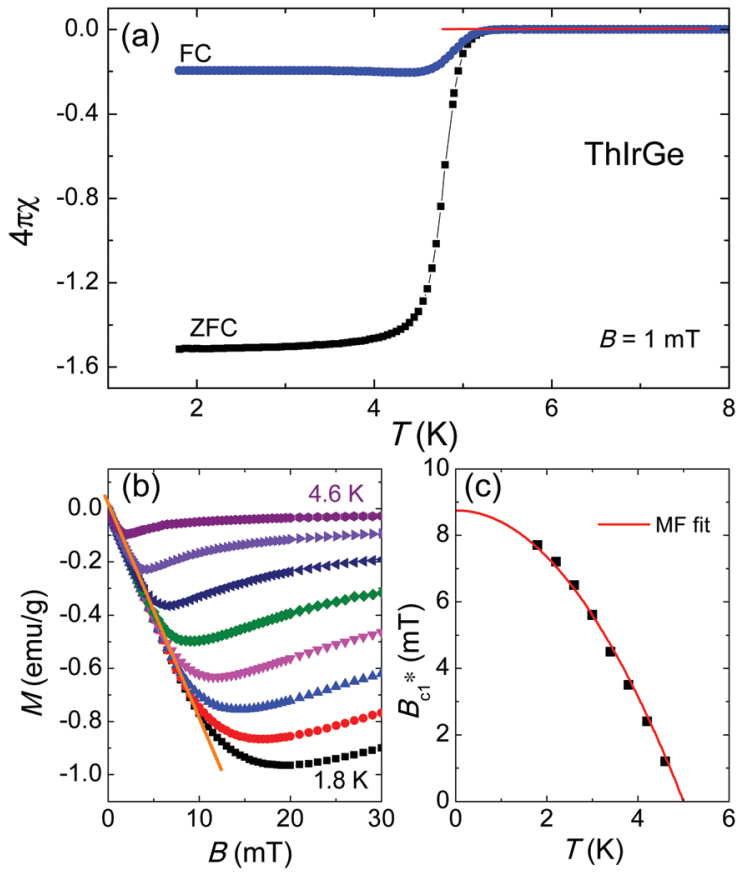

Fig. 5 (a) Low temperature magnetic susceptibility measured on the ThlrGe sample under an applied field of $1 \mathrm{mT}$. (b) Isothermal magnetization curves for the sample at various temperatures between 1.8 and $4.6 \mathrm{~K}$. The solid line denotes the initial linear behavior. (c) Temperature dependence of the effective lower critical field. The solid line is a mean-field fit to the data (see text).

which is characteristic of a type-II superconducting behavior. At $1.8 \mathrm{~K}$, the $\chi_{\mathrm{ZFC}}$ and $\chi_{\mathrm{FC}}$ values correspond to shielding and Meissner fractions of $151.5 \%$ and $19.5 \%$, respectively, without considering the demagnetization factor. Fig. 5(b) shows the isothermal magnetization curves at various temperatures between 1.8 and $4.6 \mathrm{~K}$. In the low-field region, the $M(B)$ data decrease linearly with increasing field due to the Meissner state. At each temperature, the effective lower critical field $B_{\mathrm{cl}}^{*}$ is determined as the field at which $M(B)$ deviates from its initial linearity. The resulting temperature dependence of $B_{\mathrm{cl} 1}^{*}$ is displayed in Fig. 5(c) and can be well fitted by the mean-field equation: ${ }^{32}$

$$
B_{\mathrm{c} 1}^{*}(T)=B_{\mathrm{cl}}^{*}(0)\left[1-\left(\frac{T}{T_{\mathrm{c}}}\right)^{2}\right],
$$

where $B_{\mathrm{cl}}^{*}(0)=8.8 \mathrm{mT}$ is the effective zero-temperature lower critical field. Since the demagnetization factor $N_{\mathrm{d}}$ is related to the slope of initial magnetization curve through the relation

$$
\mathrm{d} M / \mathrm{d} B=-\frac{1}{4 \pi\left(1-N_{\mathrm{d}}\right)},
$$

which gives $N_{\mathrm{d}}=0.31$. Then the zero-temperature lower critical field $B_{\mathrm{c} 1}(0)$ is calculated to be $B_{\mathrm{cl} 1}^{*}(0) /\left(1-N_{\mathrm{d}}\right)=12.8 \mathrm{mT}$. In addition, the shielding and Meissner fractions after demagnetization correction are $104.5 \%$ and $13.5 \%$, respectively, suggesting that superconductivity occurs in almost the whole sample volume. 
With the values of $B_{\mathrm{c} 1}(0)$ and $B_{\mathrm{c} 2}(0)$, various superconducting parameters can be determined. First, the Ginzburg-Landau coherence length $\xi_{\mathrm{GL}}(0)$ is calculated to be $107 \AA$ using the formula

$$
\xi_{\mathrm{GL}}(0)=\sqrt{\frac{\Phi_{0}}{2 \pi B_{\mathrm{c} 2}(0)}},
$$

where $\Phi_{0}=2.07 \times 10^{-15} \mathrm{~Wb}$ is the flux quantum. Second, the GL parameter $\kappa_{\mathrm{GL}}$ can be derived from the equation ${ }^{37}$

$$
\frac{B_{\mathrm{c} 1}(0)}{B_{\mathrm{c} 2}(0)}=\frac{\ln \kappa_{\mathrm{GL}}+0.5}{2 \kappa_{\mathrm{GL}}{ }^{2}} \text {. }
$$

This gives $\kappa_{\mathrm{GL}}$ value of 19.9 , confirming that ThIrGe is a moderate type-II superconductor. Third, the penetration depth $\lambda_{\text {eff }}$ is obtained from the formula

$$
B_{\mathrm{cl}}(0)=\frac{\Phi_{0}}{4 \pi \lambda_{\mathrm{eff}}^{2}}\left(\ln \kappa_{\mathrm{GL}}+0.5\right)
$$

which yields $\lambda_{\text {eff }}=2120 \AA$.

The bulk nature of superconductivity in ThIrGe is confirmed by the specific heat measurements. Fig. 6(a) shows the lowtemperature specific heat $\left(C_{\mathrm{p}}\right)$ results at zero field and $9 \mathrm{~T}$ plotted as $C_{\mathrm{p}} /$ T versus $T^{2}$. The zero-filed $C_{\mathrm{p}}$ data shows a distinct jump below $5.20 \mathrm{~K}$, which is very close to $T_{\mathrm{c}}$. This anomaly is
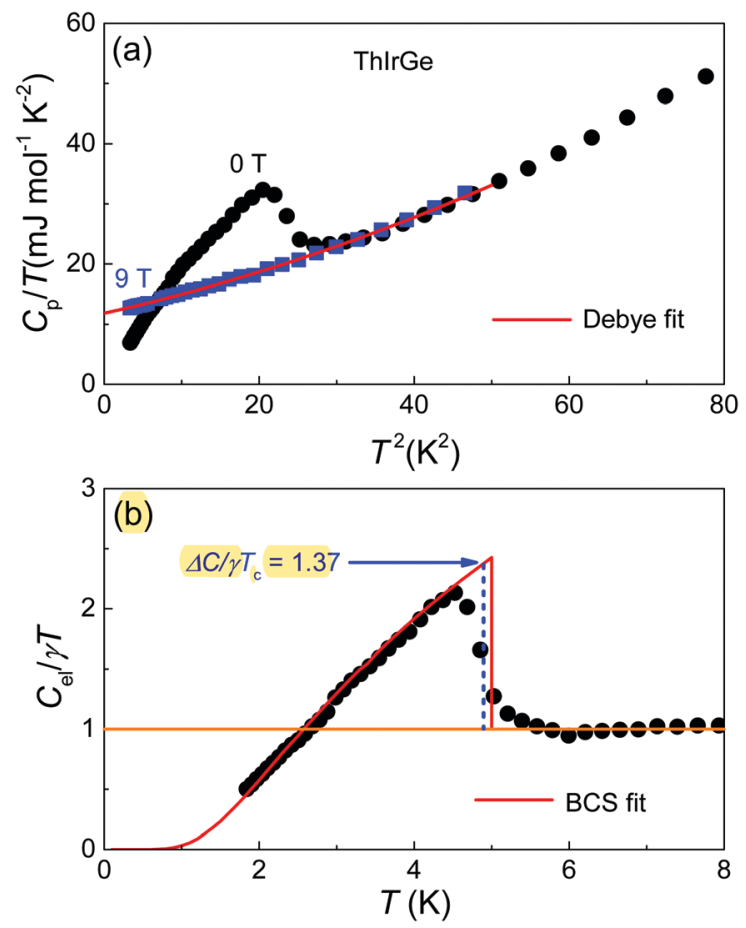

Fig. 6 (a) Low-temperature $C_{p} / T$ data measured under 0 and $9 T$ plotted as a function of $T^{2}$ for the ThlrGe sample. The red solid line is a Debye fit to the data. (b) Temperature dependence of normalized electronic specific heat at $0 \mathrm{~T}$. The red solid line is the theoretical curve from the weak coupling BCS theory. The blue dashed line and arrow demonstrate the specific heat jump $\Delta C / \gamma T_{c}$ value based on entropy conserving

\begin{tabular}{|c|c|c|}
\hline Parameter & Unit & ThIrGe \\
\hline$T_{\mathrm{c}}$ & K & 5.25 \\
\hline$B_{\mathrm{c} 1}(0)$ & $\mathrm{mT}$ & 12.8 \\
\hline$B_{\mathrm{c} 2}(0)$ & $\mathrm{T}$ & 2.9 \\
\hline$\xi_{\mathrm{GL}}(0)$ & $\AA$ & 107 \\
\hline$\lambda_{\mathrm{GL}}$ & $\AA$ & 2120 \\
\hline$\kappa_{\mathrm{GL}}$ & 一 & 19.9 \\
\hline$\gamma$ & $\mathrm{mJ} \mathrm{mol}{ }^{-1} \mathrm{~K}^{-2}$ & 11.8 \\
\hline$\beta$ & $\mathrm{mJ} \mathrm{mol}^{-1} \mathrm{~K}^{-4}$ & 0.29 \\
\hline$\Theta_{\mathrm{D}}$ & $\mathrm{K}$ & 275 \\
\hline$\Delta C / \gamma T_{\mathrm{c}}$ & - & 1.37 \\
\hline$\lambda_{\text {ep }}$ & - & 0.65 \\
\hline
\end{tabular}
construction.
Table 3 Normal-state and superconducting parameters of ThlrGe

suppressed completely under $9 \mathrm{~T}$, which allows us to analysis the normal-state data by the Debye model

$$
C_{\mathrm{p}} / T=\gamma+\beta T^{2}+\delta T^{4},
$$

where $\gamma$ is the Sommerfield coefficient and $\beta(\delta)$ is the phonon specific-heat coefficient. The best fit yields $\gamma=11.8 \mathrm{~mJ} \mathrm{~mol}^{-1} \mathrm{~K}^{-2}$, $\beta=0.29 \mathrm{~mJ} \mathrm{~mol}^{-1} \mathrm{~K}^{-4}$, and $\delta=0.0027 \mathrm{~mJ} \mathrm{~mol}^{-1} \mathrm{~K}^{-6}$. Then the Debye temperature $\Theta_{\mathrm{D}}$ is calculated to be $275 \mathrm{~K}$ using the equation

$$
\Theta_{\mathrm{D}}=\left(12 \pi^{4} N R / 5 \beta\right)^{1 / 3},
$$

where $N=3$ is the number of atoms in the formula and $R=$ $8.314 \mathrm{~J} \mathrm{~mol}^{-1} \mathrm{~K}^{-1}$ is the molar gas constant. Once $\Theta_{\mathrm{D}}$ is known, the electron-phonon coupling strength $\lambda_{\text {ep }}$ is estimated to 0.65 based on the inverted McMillan formula ${ }^{38}$

$$
\lambda_{\text {ep }}=\frac{1.04+\mu^{*} \ln \left(\Theta_{\mathrm{D}} / 1.45 T_{\mathrm{c}}\right)}{\left(1-0.62 \mu^{*}\right) \ln \left(\Theta_{\mathrm{D}} / 1.45 T_{\mathrm{c}}\right)-1.04},
$$

where $\mu^{*}=0.13$ is the Coulomb repulsion pseudopotential. Using the formula

$$
N(0)=\frac{3 \gamma}{\pi^{2} k_{\mathrm{B}}^{2}\left(1+\lambda_{\mathrm{ep}}\right)},
$$

the bare density of states at the Fermi level $N(0)$ is found to be 3.04 states $\mathrm{eV}^{-1}$ f.u. ${ }^{-1}$. After subtraction of the phonon contribution, the normalized electronic specific heat $C_{\mathrm{el}} / \gamma T$ is shown in Fig. 6(b). The entropy conversing construction of the $C_{\mathrm{p}}$ anomaly (blue dashed line) yields $\Delta C / \gamma T=1.37$, close to the BCS value of $1.43 .^{39}$ Indeed, The temperature dependence of $C_{\mathrm{el}} / \gamma T$ below the peak is well reproduced by the BCS theory with a zero-temperature gap value $\Delta(0)=0.73 \mathrm{meV}$ (red solid line). This, together with the magnitude of $\lambda_{\mathrm{ep}}$, implies that ThIrGe is a fully gapped $s$-wave superconductor in the weak coupling regime. The obtained normal-state and superconducting parameters are listed in Table 3.

\subsection{Electronic band structure}

Fig. 7(a) shows the electronic band structure for ThIrGe based on first-principles calculations both without and with SOC. In the absence of SOC, there are multiple bands crossing the Fermi level $\left(E_{\mathrm{F}}\right)$, consistent with the metallic nature of this material. Turning on the SOC results in a significant change in the band dispersion near $E_{\mathrm{F}}$. In particular, the SOC splitting 

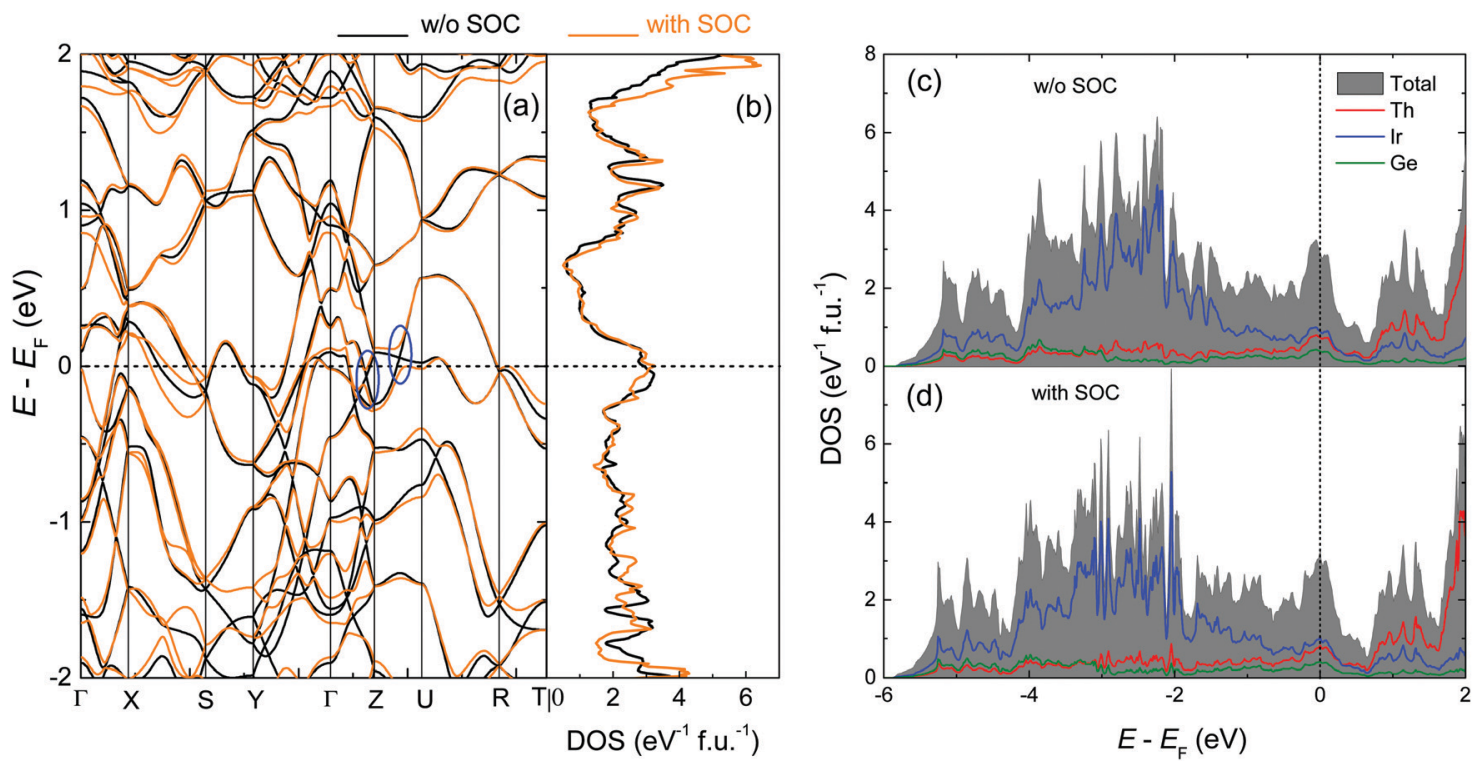

Fig. 7 (a) The electronic band structure of ThlrGe without (black) and with (orange) SOC. (b) Corresponding total density of states plotted as a function of energy. The horizontal dashed line indicates the position of Fermi level. (c and d) The projected density of states for ThlrGe without and with SOC, respectively. The vertical dashed line indicates the position of Fermi level.

reaches about $0.16 \mathrm{eV}$, and $0.17 \mathrm{eV}$ along $\Gamma-Z$ and $Z-U$ lines, respectively. As a consequence, the theoretical $N(0)$ increases from 2.91 states $\mathrm{eV}^{-1}$ f.u. ${ }^{-1}$ without SOC to 3.22 states $\mathrm{eV}^{-1}$ f.u. ${ }^{-1}$ after switching on SOC, as seen in Fig. 7(b). While the $N(0)$ from $C_{\mathrm{p}}$ measurement lies between these two values, it is obviously larger than the one without SOC, suggesting that the DOS at $E_{\mathrm{F}}$ is indeed enhanced by the SOC. The projected DOS on atomic orbitals without and with SOC are displayed in Fig. 7(c) and (d), respectively. In both cases, the DOS for $E-E_{\mathrm{F}}$ between -4 and $-1 \mathrm{eV}$ are dominated by the $5 \mathrm{~d}$ orbital of Ir atoms. Nevertheless, as $E$ moves closer to $E_{\mathrm{F}}$, the hybridization between the orbitals of $\mathrm{Th}$, Ir and Ge becomes significant. At $E_{\mathrm{F}}$, the contribution of Th orbitals is nearly equal to that of Ir orbitals and twice that of the Ge orbitals. In the $E$ range of 0.5 to $2 \mathrm{eV}$ above $E_{\mathrm{F}}$, the contribution form Th orbitals dominates over that

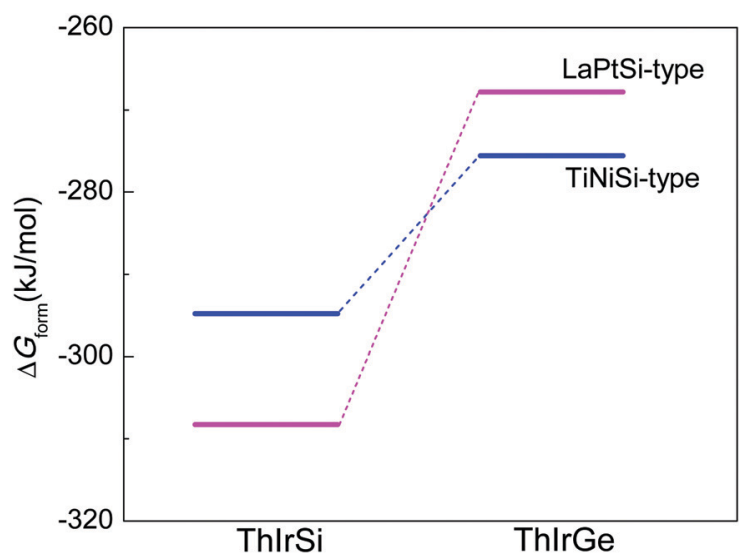

Fig. 8 The Gibbs formation energies for ThlrSi and ThlrGe in both the TiNiSi-type and LaPtSi-type structures. The two dashed lines are a guide to the eyes. from Ir orbitals while the contribution from Ge orbitals is diminished. These results clearly indicate that ThIrGe is a multiorbital system with nonnegligile spin-orbit interaction.

\subsection{Comparison between the Gibbs formation energies of ThIrGe and ThIrSi}

From the above results, it is clear that while ThIrGe is isoelectronic to ThIrSi, their crystal structures are very different. To gain insight into this issue, we examine the Gibbs formation energy $\Delta G_{\text {form }}$ of these compounds in both the TiNiSi-type and LaPtSi-type structures according to the following reaction:

$$
\operatorname{Th}(\mathrm{s})+\operatorname{Ir}(\mathrm{s})+\mathrm{Si}(\mathrm{Ge})(\mathrm{s}) \rightarrow \operatorname{ThIrSi}(\mathrm{Ge})(\mathrm{s}) .
$$

Here $\Delta G_{\text {form }}$ is obtained by evaluating the free-energy differences between the subsystems involved in the reaction, which is expressed as ${ }^{40}$

$$
\Delta G_{\text {form }}=G[\operatorname{ThIrSi}(\mathrm{Ge})]-G[\mathrm{Th}]-G[\mathrm{Ir}]-G[\mathrm{Si}(\mathrm{Ge})],
$$

where $G$ is the free energy of each subsystem. Since we deal with bulk samples, the entropy and zero-point energy effects are neglected ${ }^{41}$ and $G$ can be estimated from first principles calculations. The obtained $\Delta G_{\text {form }}$ values are summarized in Fig. 8 for better elucidation. As can be seen, $\Delta G_{\text {form }}=$ $-308.3 \mathrm{~kJ} \mathrm{~mol}^{-1}$ for ThIrSi in the LaPtSi-type structure, which is lower than that of $-294.8 \mathrm{~kJ} \mathrm{~mol}^{-1}$ in TiNiSi-type structure. The situation is opposite for ThIrGe, where $\Delta G_{\text {form }}$ is lower in the TiNiSi-type structure $\left(-275.6 \mathrm{~kJ} \mathrm{~mol}^{-1}\right)$ than in the LaPtSi-type structure $\left(-267.8 \mathrm{~kJ} \mathrm{~mol}^{-1}\right)$. These results indicate that ThIrSi and ThIrGe are energetically more stable in the LaPtSi-type and TiNiSi-type structures, respectively, in excellent agreement with the experimental observation. In this respect, it is of future 
interest to see whether $\Delta G_{\text {form }}$ can be generally applicable to predict the crystal structure of $\mathrm{MM}^{\prime} \mathrm{X}$ compounds.

\section{Conclusions}

In summary, we have synthesized and characterized a new intermetallic germanide ThIrGe. Structural analyses indicate that this compound adopts the orthorhombic TiNiSi-type structure, which is the first example of this structural type for Thbased ternary equiatomic intermetallics. Furthermore, ThIrGe is discovered to be a bulk type-II superconductor below $5.25 \mathrm{~K}$ with a BCS-like superconducting gap. Theoretical calculations reveal that a notable hybridization between the Th, Ir and Ge orbitals is present at the Fermi level and the corresponding density of states are enhanced by the spin-orbit interaction. In addition, we show that the structural difference between ThIrGe and ThIrSi is consistent with comparison between the Gibbs formation energy of these materials in both structures. Our results not only provide an isostructural nonmagnetic reference to UIrGe, but also help to better understand both the structural and superconducting behavior in the family of ternary equiatomic compounds.

\section{Conflicts of interest}

The authors declare no competing interests.

\section{Acknowledgements}

We acknowledge financial support by the foundation of Westlake University. The work at Zhejiang University is supported by National Key Research Development Program of China (No. 2017YFA0303002).

\section{Notes and references}

1 V. Johnson and W. Jeitschko, Ternary equiatomic transition metal silicides and germanides, J. Solid State Chem., 1972, 4, 123-130.

2 E. Hovestreydt, N. Engel, K. Klepp, B. Chabot and E. Parthe, Equiatomic ternary rare earth-transition metal silicides, germanides and gallides, J. Less-Common Met., 1982, 85, 247-274.

3 R. Muller, R. Shelton, J. Richardson Jr and R. Jacobson, Superconductivity and crystal structure of a new class of ternary transition metal phosphides $\mathrm{TT} / \mathrm{t}^{\prime} \mathrm{P}(\mathrm{T}=\mathrm{V}, \mathrm{Zr}, \mathrm{Nb}, \mathrm{Ta}$ and $\left.\mathrm{T} / \mathrm{t}^{\prime}=\mathrm{V}, \mathrm{Ru}, \mathrm{Rh}\right)$, J. Less-Common Met., 1983, 92, 177-183.

4 G. S. Rao, K. Wagner, G. Balakrishnan, J. Janaki, W. Paulus, R. Schollhorn, V. Subramanian and U. Poppe, Structure and superconductivity studies on ternary equiatomic silicides, MM'Si, Mater. Sci. Bull., 1985, 7, 215-228.

5 F. Canepa, P. Manfrinetti, M. Pani and A. Palenzona, Structural and transport properties of some UTX compounds where $\mathrm{T}=\mathrm{Fe}, \mathrm{Co}, \mathrm{Ni}$ and $\mathrm{X}=\mathrm{Si}, \mathrm{Ge}, J$. Alloys Compd., 1996, 234, 225-230.

6 P. R. Zhang, H. Q. Yuan and C. Cao, Electron-phonon coupling and nontrivial band topology in noncentrosymmetric superconductors LaNiSi, LaPtSi, and LaPtGe, Phys. Rev. B, 2020, 101, 245145.

7 L. Havela, M. Divis, V. Sechovsky, A. Andreev, F. Honda, G. Oomi, Y. Meresse and S. Heathman, U ternaries with ZrNiAl structure-Lattice properties, J. Alloys Compd., 2001, 322, 7-13.

8 G. A. Landrum, R. Hoffmann, J. Evers and H. Boysen, The TiNiSi family of compounds: structure and bonding, Inorg. Chem., 1998, 37, 5754-5763.

9 S. Ramakrishnan, K. Ghosh, A. D. Chinchure, V. Marathe and G. Chandra, Magnetism and superconductivity in RPtSi $(\mathrm{R}=\mathrm{La}, \mathrm{Ce}, \mathrm{Nd}$, and Sm), Phys. Rev. B: Condens. Matter Mater. Phys., 1995, 52, 6784.

10 A. O. Oliynyk, L. A. Adutwum, B. W. Rudyk, H. Pisavadia, S. Lotfi, V. Hlukhyy, J. J. Harynuk, A. Mar and J. Brgoch, Disentangling structural confusion through machine learning: structure prediction and polymorphism of equiatomic ternary phases ABC, J. Am. Chem. Soc., 2017, 139, 17870-17881.

11 G. J. Miller and J. Cheng, Ternary Metal-Rich Phosphides: Structure, Bonding, and Site Preferences in $\mathrm{ZrNbP}$ and $\mathrm{Hf}_{1+x} \mathrm{Mo}_{1-x} \mathrm{P}$, Inorg. Chem., 1995, 34, 2962-2968.

12 K. Klepp and E. Parthe, RPtSi phases (R = La, Ce, Pr, Nd, Sm and Gd) with an ordered $\mathrm{ThSi}_{2}$ derivative structure, Acta Crystallogr., Sect. B: Struct. Crystallogr. Cryst. Chem., 1982, 38, 1105-1108.

13 R. Troc and V. Tran, Magnetic properties of UT(Si,Ge) series, J. Magn. Magn. Mater., 1988, 73, 389-397.

14 T. Ueda, D. Honda, T. Shiromoto, N. Metoki, F. Honda, K. Kaneko, Y. Haga, T. D. Matsuda, T. Takeuchi, A. Thamizhavel, K. Sugiyama, K. Kindo, R. Settai and Y. Onuki, Magnetic property and pressure effect of a single crystal CeRhGe, J. Phys. Soc. Jpn., 2005, 74, 2836-2842.

15 F. Kneidinger, I. Zeiringer, A. Siderenko, E. Bauer, H. Michor, P. Rogl and J. Sereni, Physical properties of CeIrSi with trillium-lattice frustrated magnetism, Phys. Rev. $B, 2019,100,134442$.

16 W. Lee and R. Shelton, CePtSi: a new heavy-fermion compound, Phys. Rev. B: Condens. Matter Mater. Phys., 1987, 35, 5369.

17 A. Ramirez, B. Batlogg and E. Bucher, Magnetothermal properties of UIrGe and systematic trends in magnetic heavy fermion compounds, J. Appl. Phys., 1987, 61, 3189-3190.

18 W. Lee, F. Yang, C. Shih and H. Yang, Crystal structure and superconductivity in the Ni-based ternary compound LaNiSi, Phys. Rev. B: Condens. Matter Mater. Phys., 1994, 50, 6523.

19 N. Huy, A. Gasparini, D. De Nijs, Y. Huang, J. Klaasse, T. Gortenmulder, A. de Visser, A. Hamann, T. Gorlach and H. V. Lohneysen, Superconductivity on the border of weak itinerant ferromagnetism in UCoGe, Phys. Rev. Lett., 2007, 99, 067006.

20 D. Aoki, A. Huxley, E. Ressouche, D. Braithwaite, J. Flouquet, J.-P. Brison, E. Lhotel and C. Paulsen, Coexistence of 
superconductivity and ferromagnetism in URhGe, Nature, 2001, 413, 613-616.

21 Y. P. Qi, J. G. Guo, H. C. Lei, Z. W. Xiao, T. Kamiya and H. Hosono, Superconductivity in noncentrosymmetric ternary equiatomic pnictides LaMP ( $\mathrm{M}=\mathrm{Ir}$ and $\mathrm{Rh} ; \mathrm{P}=\mathrm{P}$ and As), Phys. Rev. B: Condens. Matter Mater. Phys., 2014, 89, 024517.

22 Y. Okamoto, T. Inohara, Y. Yamakawa, A. Yamakage and K. Takenaka, Superconductivity in the hexagonal ternary phosphide ScIrP, J. Phys. Soc. Jpn., 2016, 85, 013704.

23 K. Domieracki and D. Kaczorowski, Superconductivity in a non-centrosymmetric compound ThCoSi, J. Alloys Compd., 2016, 688, 206-210.

24 D. Singh, P. Biswas, A. Hillier and R. Singh, et al., Superconducting properties of the noncentrosymmetric superconductor LaPtGe, Phys. Rev. B, 2018, 98, 214505.

25 T. Hattori, Y. Ihara, Y. Nakai, K. Ishida, Y. Tada, S. Fujimoto, N. Kawakami, E. Osaki, K. Deguchi, N. Sato and I. Satoh, Superconductivity induced by longitudinal ferromagnetic fluctuations in UCoGe, Phys. Rev. Lett., 2012, 108, 066403.

26 B. Chevalier, B. Lloret, P. Gravereau, B. Buffat and J. Etourneau, Kondo fluctuations and magnetism in the new equiatomic ternary compounds UIrSi and UIrGe, J. Magn. Magn. Mater., 1988, 75, 13-18.

27 P. Manfrinetti, A. Palenzona, S. Dhar and C. Mitra, Structure and properties of some new Th compounds, Intermetallics, 1999, 7, 1291-1294.

28 A. Ptok, K. Domieracki, K. Kapcia, J. Lazewski, P. Jochym, M. Sternik, P. Piekarz and D. Kaczorowski, Electronic and lattice properties of noncentrosymmetric superconductors ThTSi (T = Co, Ir, Ni, and Pt), Phys. Rev. B, 2019, 100, 165130.

29 B. H. Toby, EXPGUI, a graphical user interface for GSAS, J. Appl. Crystallogr., 2001, 34, 210-213.

30 G. Kresse and J. Furthmuller, Efficiency of ab-initio total energy calculations for metals and semiconductors using a plane-wave basis set, Comput. Mater. Sci., 1996, 6, 15-50.
31 J. P. Perdew, K. Burke and M. Ernzerhof, Generalized gradient approximation made simple, Phys. Rev. Lett., 1996, 77, 3865.

32 G. R. Xiao, S. Q. Wu, B. Z. Li, B. Liu, J. F. Wu, Y. W. Cui, Q. Q. Zhu, G. H. Cao and Z. Ren, Normal-state and superconducting properties of the cubic Laves phase $\mathrm{ThIr}_{2}$, Intermetallics, 2021, 128, 106993.

33 T. Geballe, B. Matthias, V. Compton, E. Corenzwit, G. Hull Jr and L. D. Longinotti, Superconductivity in binary alloy systems of the rare earths and of thorium with Pt-group metals, Phys. Rev., 1965, 137, A119.

34 X. Wang, C. B. J. Etourneau and P. Hagenmuller, Crystal structure and superconductivity of new ternary MTGe germanides ( $\mathrm{M}=\mathrm{Ti}, \mathrm{Zr}$, Hf and $\mathrm{T}=\mathrm{Ru}, \mathrm{Os}, \mathrm{Rh}, \mathrm{Ir})$, Mater. Res. Bull., 1987, 22, 331.

35 N. Werthamer, E. Helfand and P. Hohenberg, Temperature and purity dependence of the superconducting critical field, $\mathrm{Hc}_{2}$. III. Electron spin and spin-orbit effects, Phys. Rev., 1966, 147, 295.

36 A. M. Clogston, Upper limit for the critical field in hard superconductors, Phys. Rev. Lett., 1962, 9, 266.

37 C.-R. Hu, Numerical constants for isolated vortices in superconductors, Phys. Rev. B: Condens. Matter Mater. Phys., 1972, 6, 1756.

38 W. McMillan, Transition temperature of strong-coupled superconductors, Phys. Rev., 1968, 167, 331.

39 J. Bardeen, L. N. Cooper and J. R. Schrieffer, Therory of Superconductivity, Phys. Rev., 1957, 108, 1175.

40 J. I. Martinez, H. A. Hansen, J. Rossmeisl and J. K. Norskov, Formation energies of rutile metal dioxides using density functional theory, Phys. Rev. B: Condens. Matter Mater. Phys., 2009, 79, 045120.

41 M. Bollinger, K. W. Jacobsen and J. K. Norskov, Atomic and electronic structure of $\mathrm{MoS}_{2}$ nanoparticles, Phys. Rev. B: Condens. Matter Mater. Phys., 2003, 67, 085410. 